\title{
Adaptive POD-DEIM model reduction based on an improved error estimator
}

\section{Sridhar Chellappa ${ }^{1}$, Lihong Feng ${ }^{1}$, Peter Benner ${ }^{1}$}

${ }^{1}$ Max Planck Institute for Dynamics of Complex Technical Systems, Computational Methods in Systems and Control Theory, Sandtorstraße 1, Magdeburg, Germany.

\section{Model reduction for nonlinear dynamical systems}

For reliable, efficient, rapid simulations of dynamical systems, a reduced order model (ROM) with certified accuracy is highly desirable. The ROM is derived from a full order model (FOM) through model reduction. In this work we propose an adaptive approach for nonlinear model reduction by making use of a suitable output error estimator, thus enable the generation of a compact ROM, with appropriate balance between the state and nonlinear approximations.

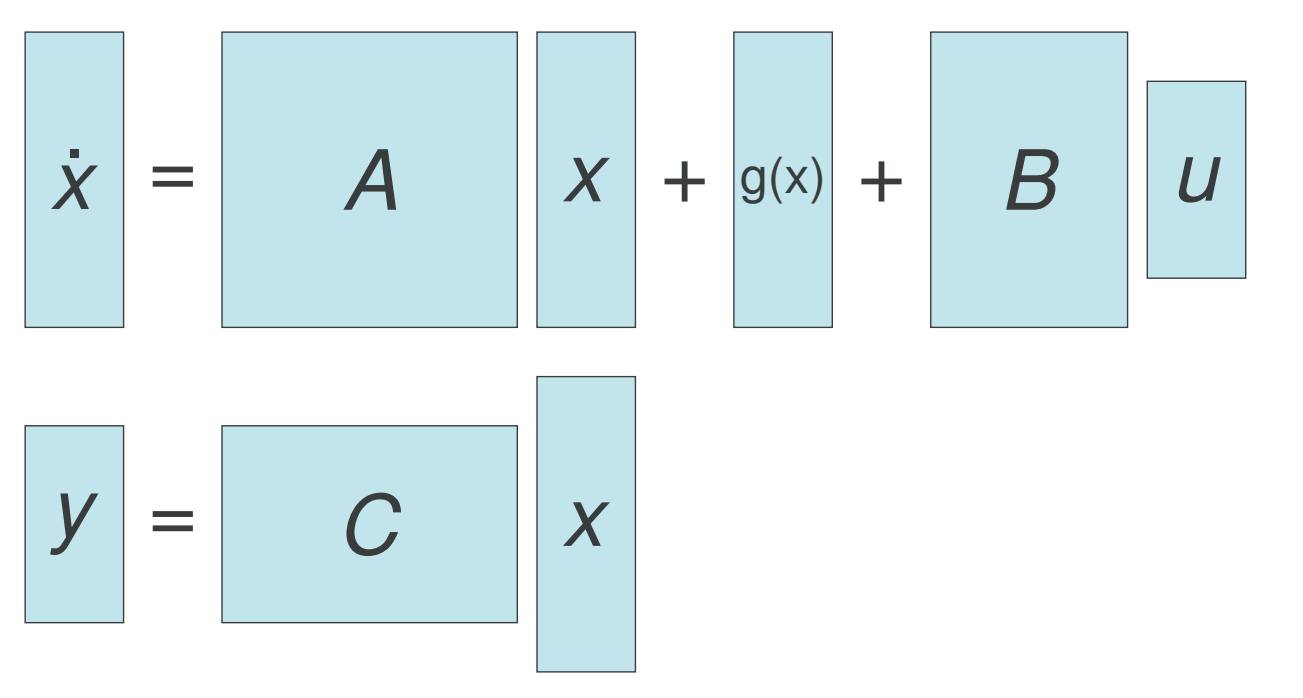

- $x \in \mathbb{R}^{n}$ - state vector,

- $u \in \mathbb{R}^{m}$ - input vector,

- $y \in \mathbb{R}^{p}$ - output vector,
- $g(.) \in \mathbb{R}^{n}$ - nonlinear term

- $\hat{g}(.) \in \mathbb{R}^{n}$ - approximated nonlinea

$g(.) \in$

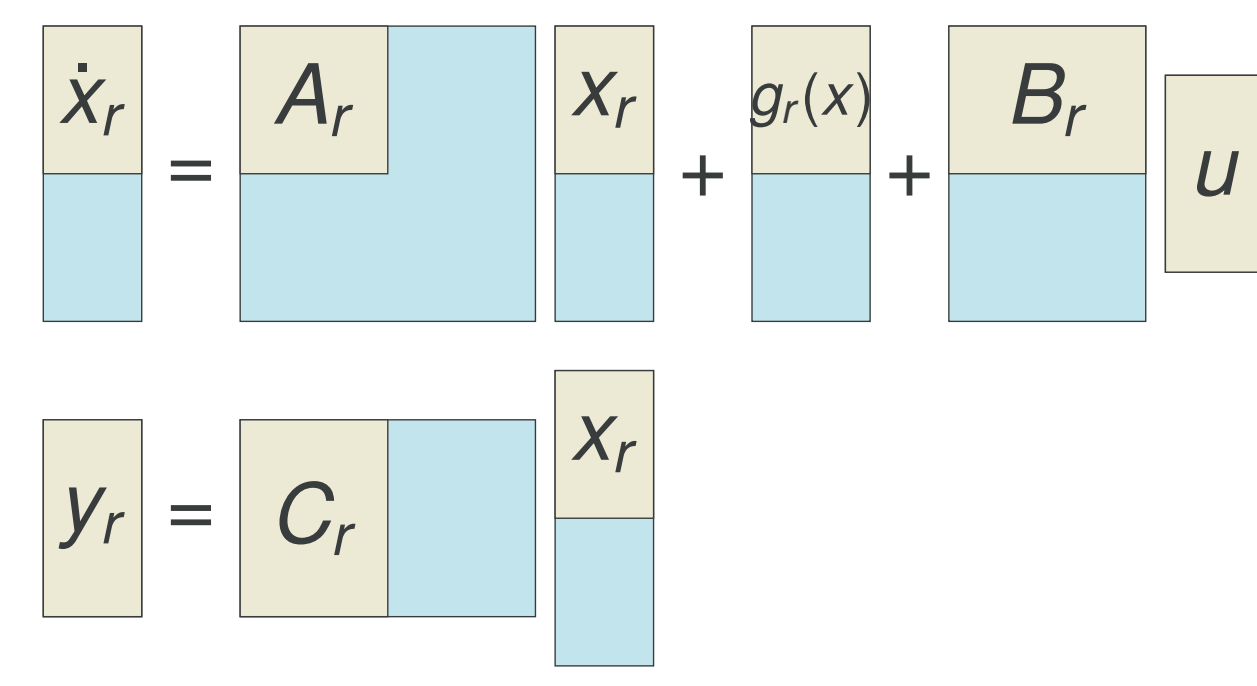

- $A \in \mathbb{R}^{n \times n}$ - state matrix

- $A \in \mathbb{R}^{n \times m}$ - input matrix

- $C \in \mathbb{R}^{p \times n}$ - output matrix,

- $V \in \mathbb{R}^{n \times n}$ - output matrix,

- $A_{r}, B_{r}, C_{r}, x_{r}, y_{r}, g_{r}$ - reduced quan$A_{r}, B_{r}, C_{r}, x_{r}, y_{r}, g_{r}-r e d u c e$
tities and $g_{r}():.=V^{\top} \widehat{g}($.$) .$

\section{Snapshots-based model reduction}

Proper Orthogonal Decomposition (POD)

Snapshots matrix $X=\left[x\left(t_{1}\right) x\left(t_{2}\right) \ldots x\left(t_{k}\right)\right] \stackrel{S V D}{\longrightarrow}=M \Sigma R^{T}$

- Projection matrix $V=M(:, 1: r)$. $r$ chosen from the decay of singular values such that,

- $\frac{\sum_{i=r+1}^{i=n} \sigma_{i}}{\sum_{i=1}^{i=r} \sigma_{i}}<$ tolerance

Drawback: If only some output quantity is of interest, such an approach is very conservative. Also, it is not possible, a priori, to determine the value of $r$, such that the ROM respects some predefined tolerance.

Remedy: An adaptive approach, making use of an appropriate error estimator (see next section).

Discrete Empirical Interpolation Method (DEIM)

- POD leads to dimension reduction but no complexity reduction for nonlinear term.

- [1] proposes a method to reduce the complexity.

Nonlinear term snapshots matrix $G=\left[g\left(x\left(t_{1}\right)\right) g\left(x\left(t_{2}\right)\right) \ldots g\left(x\left(t_{k}\right)\right)\right] \stackrel{S V D}{\longrightarrow} J \Sigma_{\text {deim }} L^{T}$

- DEIM projection matrix $U_{\text {deim }}=J(:, 1: \ell), \widehat{g}(x) \approx U_{\text {deim }}$

- $\ell$ chosen the same way as done for $r$ in the POD case above.

\section{A posteriori error estimation}

Choose $r, \ell$ adaptively using an a posteriori error estimator

- Discretization: Finite volume/Finite difference method in space and Semi-implicit Euler method in time.

- Primal-dual formulation for improved convergence and effectivity [2, 3, 4].

Primal system: $E_{d} x^{k+1}=A_{d} x^{k}+\Delta t \widehat{g}\left(x^{k}\right)+\Delta t B u^{k} \quad$ Dual system: $E_{d}^{T} x_{d u}^{k+1}=-C^{T}$

Error estimator

$$
\Delta:=\left\|y^{k+1}-\widehat{y}^{k+1}\right\| \leq \phi^{k+1}\left\|r_{p r}^{k+1}\right\|
$$

where, $\phi^{k+1}:=S^{k+1}\left(\left\|E_{d}^{-1}\right\|\left\|r_{d u}\right\|+\left\|\widehat{x}_{d u}\right\|\right), S^{k+1}:=\frac{\left\|\tilde{r}^{k+1}\right\|}{\left\|r^{k+1}\right\|}$

Improved error estimato

To achieve better effectivity, we consider the corrected output given as, $\bar{y}=\widehat{y}-\widehat{x}_{d u}^{T} r_{p r}$

$$
\text { where, } \bar{\Delta}:=\left\|y^{k+1}-\bar{y}^{k+1}\right\| \leq \omega^{k+1}\left\|r_{p r}^{k+1}\right\|
$$

$\omega^{k+1}:=S^{k+1}\left\|E_{d}^{-1}\right\|\left\|r_{d u}\right\|+\left|1-S^{k+1}\right|\left\|\widehat{x}_{d u}\right\|$

Note: The primal system residual can be split to give the POD and DEIM error contributions separately, i.e. $r_{p r}^{k+1}=r_{D \in M}^{k+1}+e_{D E M M}^{k}$. Therefore, the error estimator itself can be separated into POD and DEIM contributions as, $\triangle_{P O D}, \Delta_{D E I M}$.

\section{Adaptivity}

- Start with initial $r_{0}$ and $\ell_{0}$ columns for POD and DEIM projection matrices.

- Iteratively determine number of additional columns to be added/deleted.

$$
\begin{aligned}
& r^{i+1}=r^{i}+\Delta r, \text { with } \Delta r= \pm 1+\log _{10}\left(\frac{\Delta_{P O D}}{\text { tolerance }}\right) \\
& \ell^{i+1}=\ell^{i}+\Delta \ell, \text { with } \Delta \ell= \pm 1+\log _{10}\left(\frac{\Delta_{D E I M}}{\text { tolerance }}\right)
\end{aligned}
$$

- OFFLINE: Snapshot collection and generation of conservative POD and DEIM projection matrices.

- ONLINE: Adaptive process to determine an optimal POD and DEIM basis combination, from the offline matrices, respecting the required output error tolerance.

To avoid the stagnation of the iteration process (visiting same POD-DEIM basis combination more than once, or cycling repeatedly between a certain set of combinations), we incorporate a simple mechanism.

Idea:

- Store the POD/DEIM basis visited already in an array. Check if any combination is revisited, at each iteration. If so, add/subtract by one and recheck.

- Store the sign of the update at each iteration $( \pm 1)$ in an array. At each iteration, determine the direction of the process as the sign of the majority of the past updates.

\section{Results}

Burgers' Equation

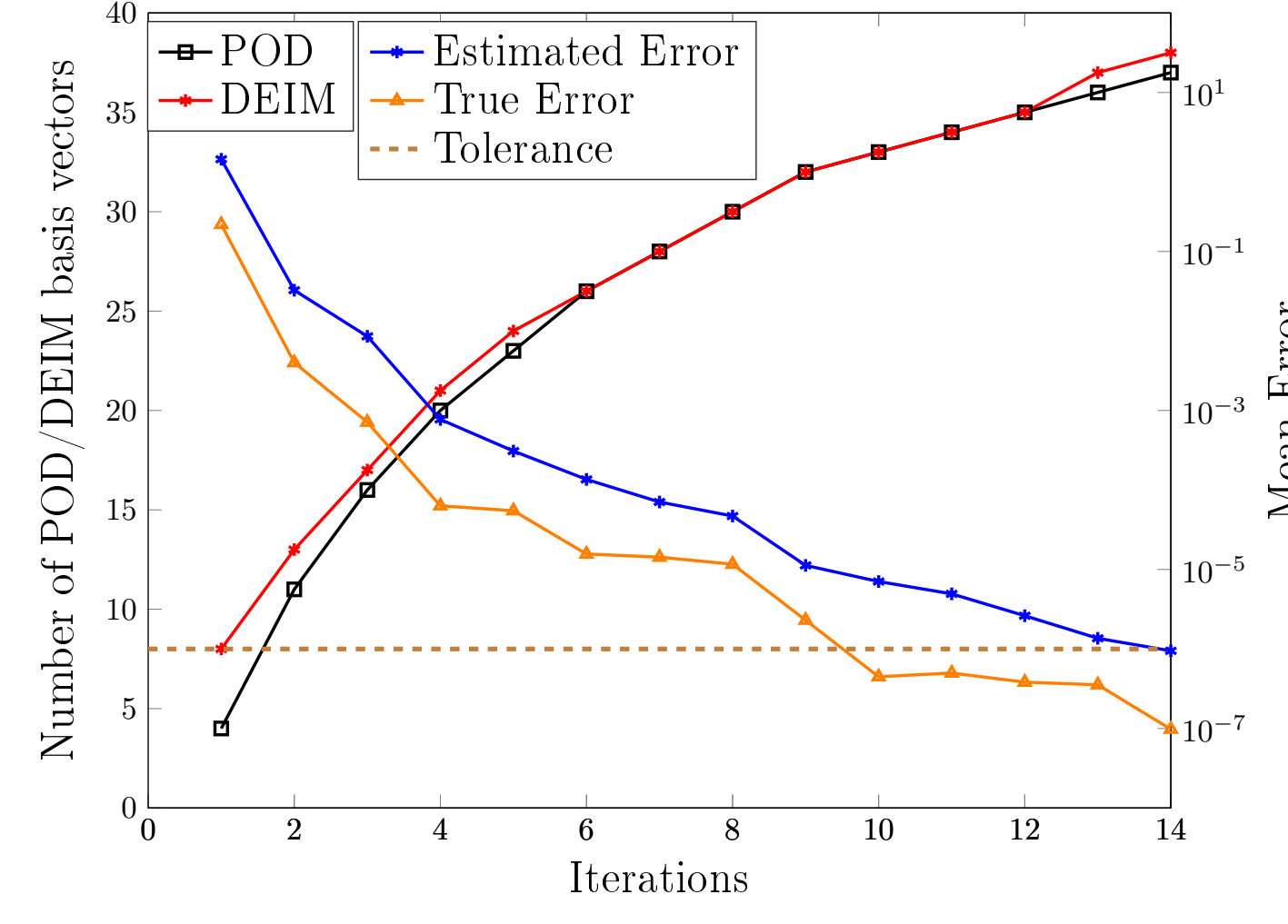

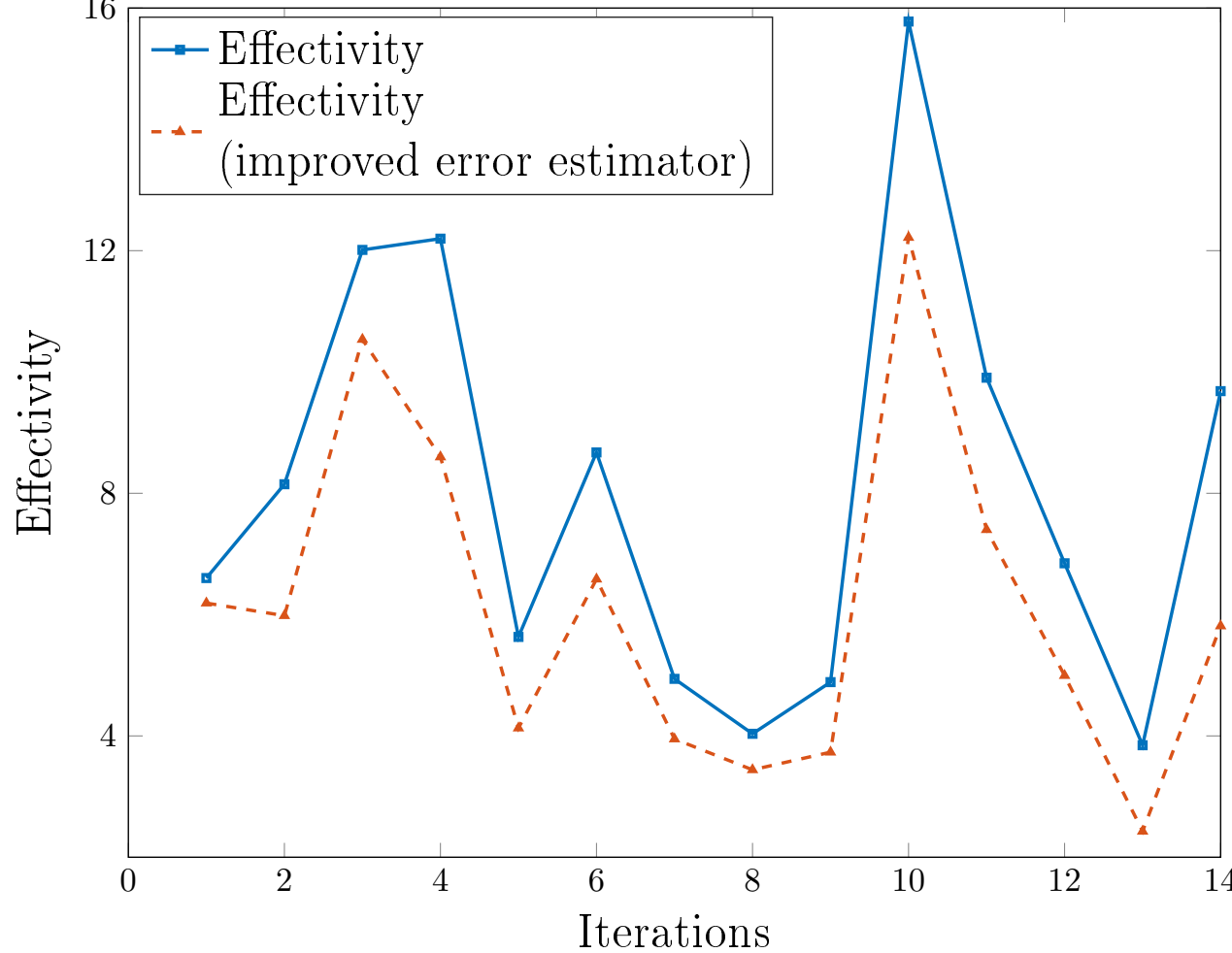

(b)
Figure 1: Adaptivity for Burgers' equation

\begin{tabular}{l|l|l|l|l}
$n$ (FOM) & $r_{0}$ & $\ell_{0}$ & $r$ (ROM) & $\ell$
\end{tabular}

\begin{tabular}{l|l|l|l|l}
1000 & 4 & 8 & 37 & 38
\end{tabular}

Table 1: Data for adaptive algorithm for Burgers' equation

Fluidized Bed Crystallizer (FBC)

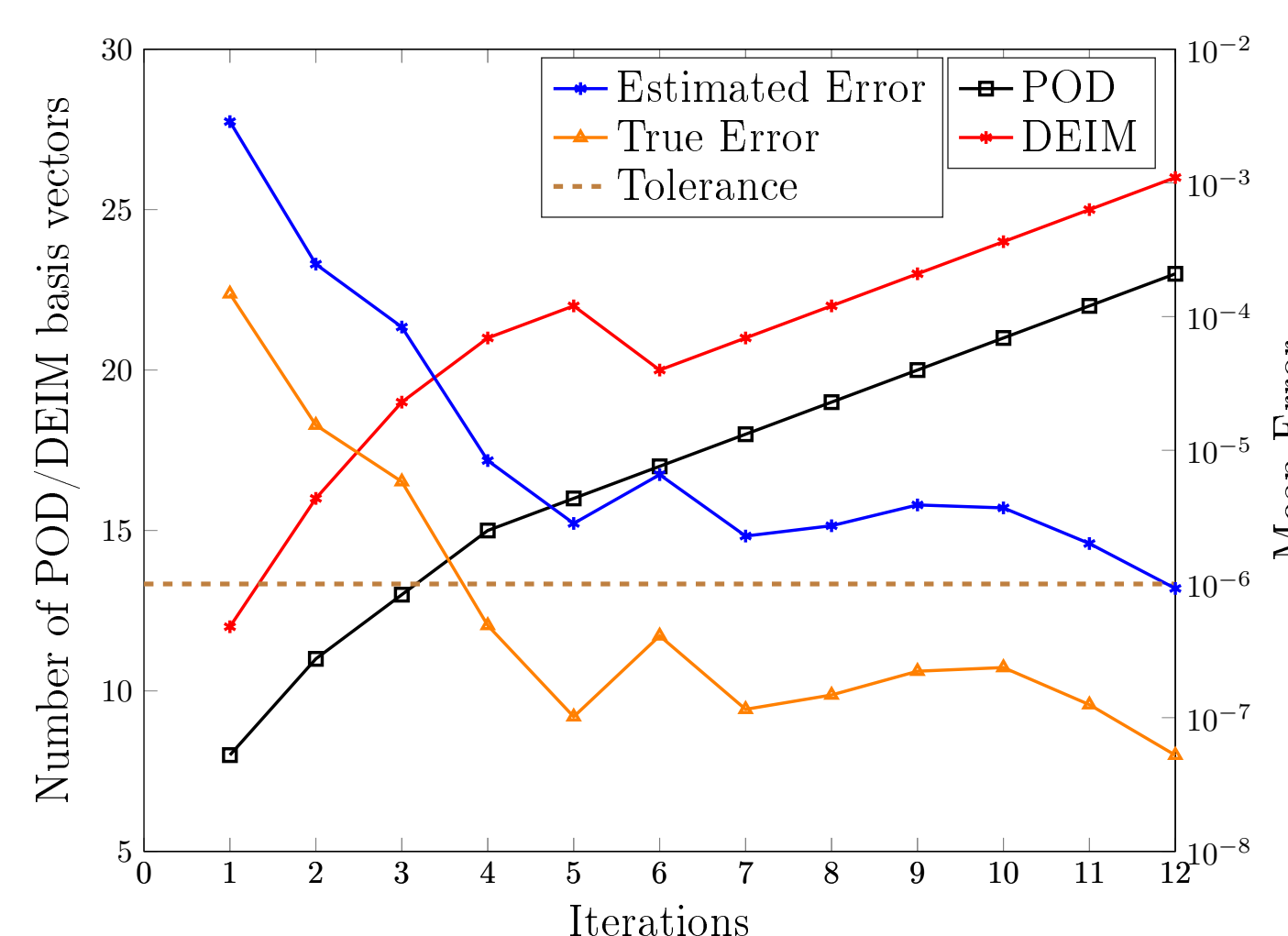

(a)

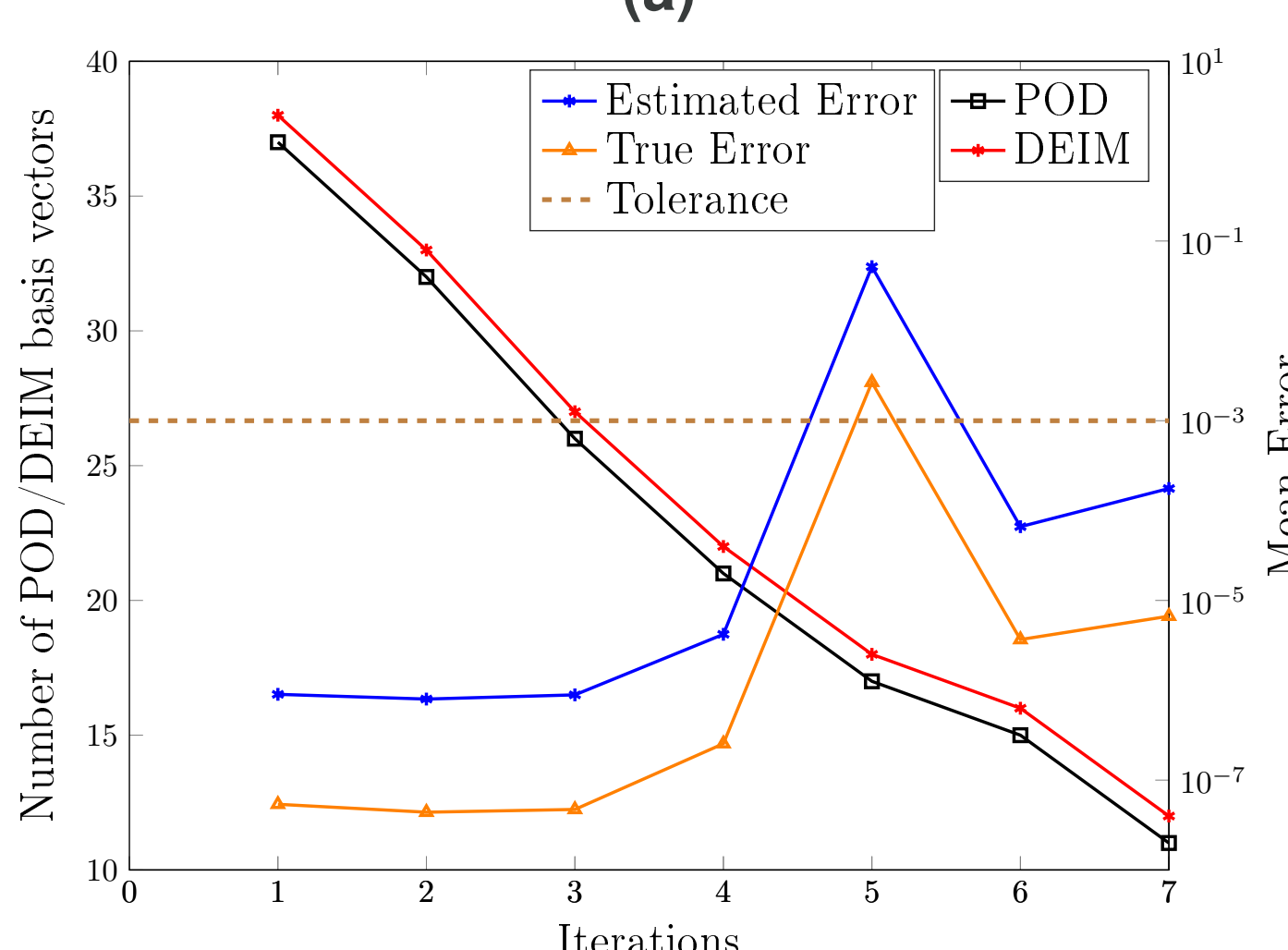

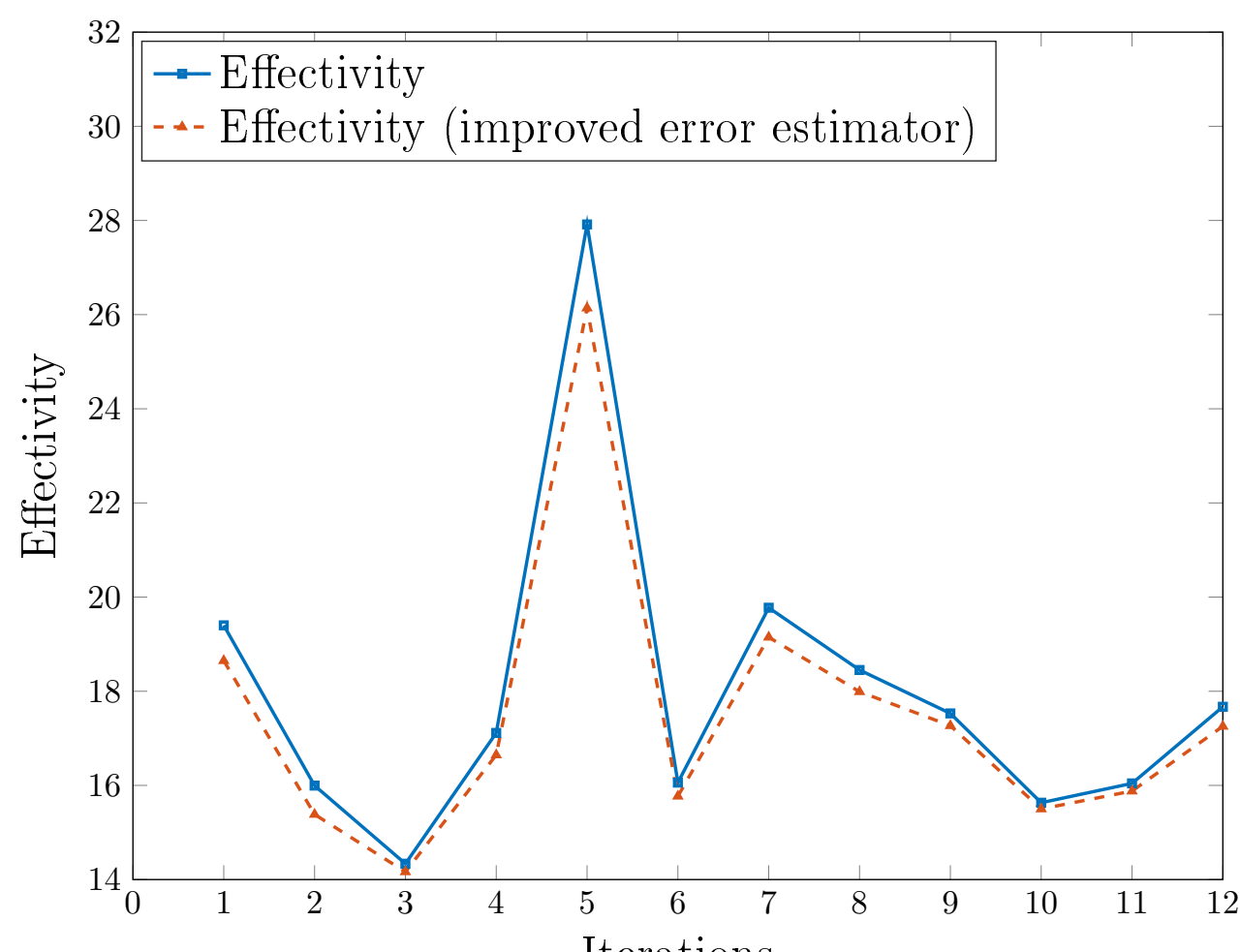

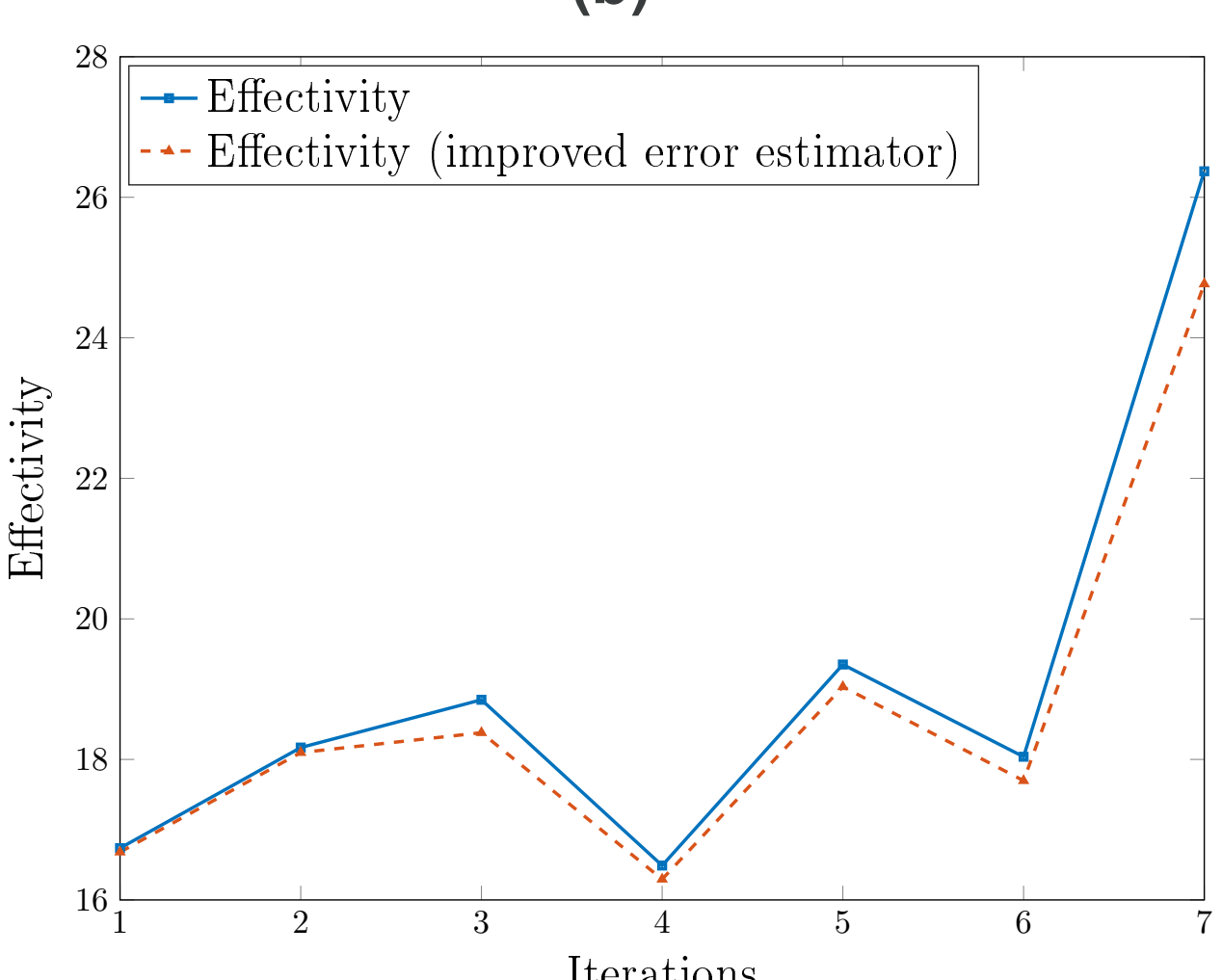

(d)
Figure 2: Adaptivity for the FBC - (a) (b) show increasing the basis adaptively from a small $r_{0}$ and $l_{0}$ (c), (d) show decreasing the basis adaptively from a large $r_{0}$ and $f_{0}$

\begin{tabular}{c|c|c|c|c|c} 
Mode & $n(\mathrm{FOM})$ & $r_{0}$ & $\ell_{0}$ & $r(\mathrm{ROM})$ & $\ell$ \\
Increasing & 18400 & 8 & 12 & 23 & 26 \\
Decreasing & 18400 & 37 & 38 & 11 & 12
\end{tabular}

Table 2: Data for adaptive algorithm for the FBC

\section{Outlook}

Extend the adaptivity idea for parametric systems through the POD-Greedy algorithm

\section{References}

[1] S. Chaturantabut AND D. C. Sorensen, Nonlinear model reduction via discrete empirical interpolation, SIAM J. Sci. Comput., 32 (2010), pp. 2737-2764.

2] L. FENG, A. C. ANTOULAS, AND P. BENNER, Some a posteriori error bounds for reduced order modelling of (non-)parametrized linear systems, ESAIM: M2AN, (2017)

[3] L. Feng, M. MANGOLD, AND P. BENNER, Adaptive POD-DEIM basis construction and its application to a nonlinear population balance system, AIChE J., (2017).

[4] Y. ZHANG, L. FENG, S. LI, AND P. BENNER, An efficient output error estimation for model order reduction of parametrized evolution equations, SIAM J. Sci. Comput., 37 (2015), pp. B910 B936. 